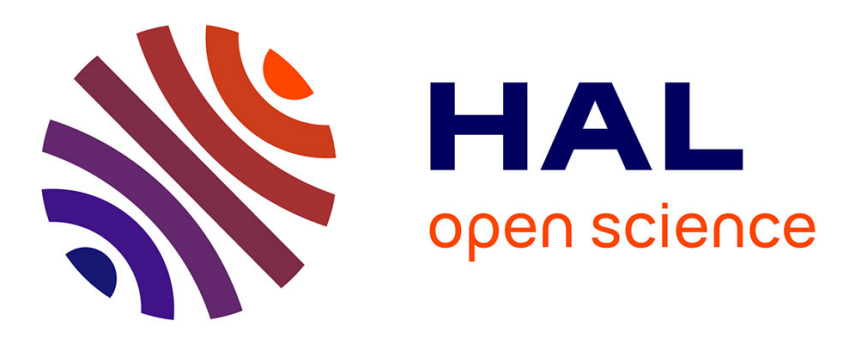

\title{
An error analysis in the algebraic estimation of a noisy sinusoidal signal
}

Da-Yan Liu, Olivier Gibaru, Wilfrid Perruquetti, Michel Fliess, Mamadou Mboup

\section{- To cite this version:}

Da-Yan Liu, Olivier Gibaru, Wilfrid Perruquetti, Michel Fliess, Mamadou Mboup. An error analysis in the algebraic estimation of a noisy sinusoidal signal. 16th Mediterranean Conference on Control and Automation, Jun 2008, Ajaccio, France. inria-00300234

\section{HAL Id: inria-00300234 \\ https://hal.inria.fr/inria-00300234}

Submitted on 17 Jul 2008

HAL is a multi-disciplinary open access archive for the deposit and dissemination of scientific research documents, whether they are published or not. The documents may come from teaching and research institutions in France or abroad, or from public or private research centers.
L'archive ouverte pluridisciplinaire HAL, est destinée au dépôt et à la diffusion de documents scientifiques de niveau recherche, publiés ou non, émanant des établissements d'enseignement et de recherche français ou étrangers, des laboratoires publics ou privés. 


\title{
An error analysis in the algebraic estimation of a noisy sinusoidal signal
}

\author{
Dayan Liu ${ }^{1}$, Olivier Gibaru ${ }^{2}$, Wilfrid Perruquetti ${ }^{1}$, Michel Fliess ${ }^{3}$, Mamadou Mboup ${ }^{4}$ \\ ${ }^{1}$ LAGIS \& INRIA-ALIEN, Ecole Centrale de Lille, 59845 Villeneuve d'Ascq, France \\ dayan.liu@inria.fr Wilfrid.perruquetti@ec-lille.fr \\ ${ }^{2}$ Arts \& Métiers ParisTech \& INRIA-ALIEN, 8 Boulevard Louis XIV, 59046 Lille, France \\ Olivier.gibaru@ensam.eu \\ ${ }^{3}$ LIX (CNRS-UMR 7161) \& INRIA-ALIEN, École Polytechnique, 91128 Palaiseau, France \\ Michel.Fliessepolytechnique.fr \\ ${ }^{4}$ UFR Math-Info \& INRIA-ALIEN, Université Paris Descartes, 75270 Paris cedex 06, France \\ Mamadou.Mboup@math-info.univ-paris5.fr
}

\begin{abstract}
The classic example of a noisy sinusoidal signal permits for the first time to derive an error analysis for a new algebraic and non-asymptotic estimation technique. This approach yields a selection of suitable parameters in the estimation procedure, in order to minimize the noise corruption.
\end{abstract}

\section{INTRODUCTION}

Recent algebraic parametric estimation techniques for linear systems [4], [5] have been extended to various problems in signal processing (see, e.g., [3], [6], [7], [8], [10], [11], [12]). Let us emphasize that those methods, which are algebraic and non-asymptotic, exhibit good robustness properties with respect to corrupting noises, without the need of knowing their statistical properties ${ }^{1}$. Nevertheless, when compared to classical approaches to communication engineering (see, e.g., [9]), a weakness of the above methods was a lack of any precise error analysis, when they were implemented in practice. The aim of this paper is to start such an analysis via the estimation of a sinusoidal signal, corrupted by an additive white Gaussian noise, where the sampling which is necessary for performing the numerical simulations is taken into account. One of the main advantages of the above mentioned algebraic estimation methods is that they provide explicit formula for the each estimate. These formulae are given in terms of iterated integrals of the observation signal.

In Sect. II, we apply the trapezoidal rule in order to compute the integrals in the algebraic estimation methods. Consequently, each estimation values of the parameters in the integrals are random Gaussian variables, whose mean and variance are easy to calculate. The estimation error due to the Gaussian noise, can be obtained with a given probability. It allows to optimize the selection of the parameters of our algebraic methods so that the approximation of $\omega$ (resp. $x_{0}$ the initial value of a real valued signal $x$ and $\dot{x}_{0}$ the initial value of $\dot{x}$ ) can be calculated with a given error, or with a minimum number values of the noisy signal.

\footnotetext{
${ }^{1}$ See [1], [2] for more theoretical details. The robustness properties have already been confirmed by numerous computer simulations and several laboratory experiments.
}

In Sect. III, we introduce a new method. The expressions of the errors due to the Gaussian noise, so obtained, are more complicated than in the previous method. In order to simplify the calculations, we assume that $\omega$ is known. Consequently, we can estimate $A$ and $\phi$ with the same method than in the previous section.

\section{Algebraic IDENTIFICATION TECHNiQUeS}

\section{A. Some useful formulae}

Proposition 1: Let $y(t)=x(t)+n(t)$ be a noisy observation on a finite time interval of a real valued signal $x$, where $x(t)=A \sin (\omega t+\phi)$. Using some algebraic techniques, the parameters $\omega, A$ and $\phi$ can be estimated from the noisy observation $y(t), t \in[0, T]$ by:

$$
\begin{aligned}
\tilde{\omega} & =\left(\frac{\int_{0}^{1} p_{1}(\tau) y(T \tau) d \tau}{\int_{0}^{1} p_{2}(\tau) y(T \tau) d \tau}\right)^{\frac{1}{2}}, \\
\tilde{A} & =\left(\tilde{x}_{0}^{2}+\frac{\tilde{\dot{x}}_{0}^{2}}{\tilde{\omega}^{2}}\right)^{\frac{1}{2}}, \\
\tilde{\phi} & =\arctan \left(\tilde{\omega} \frac{\tilde{x}_{0}}{\tilde{\dot{x}}_{0}}\right),
\end{aligned}
$$

where $\quad \tilde{x}_{0}=\int_{0}^{1} p_{3}(\tau) y(T \tau) d \tau ; \quad \tilde{\dot{x}}_{0}=\int_{0}^{1} p_{4}(\tau) y(T \tau) d \tau$,

$$
\begin{aligned}
p_{1}(\tau)= & \sum_{i=k-2}^{k}\left(\begin{array}{c}
k \\
i
\end{array}\right) \frac{2}{(2+i-k) !} \frac{(-1)^{i+1}}{(\mu+k-3-i) !}(1-\tau)^{\mu+k-3-i} \tau^{i}, \\
p_{2}(\tau)= & T^{2} \frac{(-1)^{k}}{(\mu-1) !}(1-\tau)^{\mu-1} \tau^{k} \\
p_{3}(\tau)= & -(\mu-1)(\mu-2)(1-\tau)^{\mu-3} \tau+ \\
& 2(\mu-1)(1-\tau)^{\mu-2}-\tilde{\omega}^{2} T^{2}(1-\tau)^{\mu-1} \tau, \\
p_{4}(\tau)= & p(\tau)-\frac{\mu-1}{T} p_{3}(\tau) \\
p(\tau)= & \frac{(\mu-1)(\mu-2)}{T}(1-\tau)^{\mu-3}+\tilde{\omega}^{2} T(1-\tau)^{\mu-1}
\end{aligned}
$$

Proof: Given the harmonic oscillator equation $\ddot{x}(t)+$ $\omega^{2} x(t)=0$, by applying the Laplace transform, one obtains

$$
s^{2} \hat{x}(s)-s x_{0}-\dot{x}_{0}+\omega^{2} \hat{x}(s)=0 .
$$


Take the $k(k \geq 2)$ times derivatives of both sides with respect to $s$ :

$$
\sum_{i=k-2}^{k}\left(\begin{array}{l}
k \\
i
\end{array}\right) \frac{2}{(2+i-k) !} s^{2+i-k} \hat{x}^{(i)}(s)+\omega^{2} \hat{x}^{(k)}(s)=0 .
$$

Then, multiply both sides by $s^{-\mu}, \mu \geq 3$ :

$$
\frac{\omega^{2}}{s^{\mu}} \hat{x}^{(k)}(s)=-\sum_{i=k-2}^{k}\left(\begin{array}{l}
k \\
i
\end{array}\right) \frac{2}{(2+i-k) !} \frac{1}{s^{\mu+k-2-i}} \hat{x}^{(i)}(s) .
$$

Then, express (5) back in the time domain and denote by $T$ the estimation time:

$$
\begin{gathered}
\omega^{2} \frac{(-1)^{k}}{(\mu-1) !} \int_{0}^{T}(T-\tau)^{\mu-1} \tau^{k} x(\tau) d \tau= \\
-\sum_{i=k-2}^{k}\left(\begin{array}{c}
k \\
i
\end{array}\right) \frac{2}{(2+i-k) !} \frac{(-1)^{i}}{(\mu+k-3-i) !} \int_{0}^{T}(T-\tau)^{\mu+k-3-i} \tau^{i} x(\tau) d \tau .
\end{gathered}
$$

Finally, replace $x(\tau)$ by the noisy observation $y(\tau)$ :

$$
\tilde{\omega}=\left(\frac{\int_{0}^{1} p_{1}(\tau) y(T \tau) d \tau}{\int_{0}^{1} p_{2}(\tau) y(T \tau) d \tau}\right)^{\frac{1}{2}}
$$

Now, in order to compute an estimate for $x_{0}$, one takes the derivative of both sides of (4) with respect to $s$ :

$$
s^{2} \hat{x}^{\prime}(s)+2 s \hat{x}(s)-x_{0}+\omega^{2} \hat{x}^{\prime}(s)=0 .
$$

Then, multiply both sides by $s^{-\mu}, \mu \geq 3$, yielding to:

$$
\frac{1}{s^{\mu-2}} \hat{x}^{\prime}(s)+\frac{2}{s^{\mu-1}} \hat{x}(s)-\frac{1}{s^{\mu}} x_{0}+\frac{\omega^{2}}{s^{\mu}} \hat{x}^{\prime}(s)=0 .
$$

Then, express (6) back in the time domain and denote by $T$ the estimation time:

$$
\begin{gathered}
\int_{0}^{T}\left(\frac{-1}{(\mu-3) !}(T-\tau)^{\mu-3} \tau+\frac{2}{(\mu-2) !}(T-\tau)^{\mu-2}\right) x(\tau) d \tau \\
-\frac{T^{\mu-1}}{(\mu-1) !} x_{0}-\frac{\omega^{2}}{(\mu-1) !} \int_{0}^{T}(T-\tau)^{\mu-1} \tau x(\tau) d \tau=0
\end{gathered}
$$

Finally, replace $x(\tau)$ by $y(\tau)$ to obtain an estimate for $x_{0}$ :

$$
\tilde{x}_{0}=\int_{0}^{1} p_{3}(\tau) y(T \tau) d \tau
$$

Now, to compute an estimate for $\dot{x}_{0}$, multiply both sides of (4) by $s^{-\mu}, \mu \geq 3$ :

$$
\frac{1}{\mu-2} \hat{x}(s)-\frac{1}{\mu-1} x_{0}-\frac{1}{\mu} \dot{x}_{0}+\frac{\omega^{2}}{\mu} \hat{x}(s)=0 .
$$

Then, express (8) back in the time domain and denote by $T$ the estimation time:

$$
\begin{array}{r}
\frac{1}{(\mu-3) !} \int_{0}^{T}(T-\tau)^{\mu-3} x(\tau) d \tau-\frac{T^{\mu-2}}{(\mu-2) !} x_{0} \\
-\frac{T^{\mu-1}}{(\mu-1) !} \dot{x}_{0}+\frac{\omega^{2}}{(\mu-1) !} \int_{0}^{T}(T-\tau)^{\mu-1} x(\tau) d \tau=0 .
\end{array}
$$

Replace $x(\tau)$ by $y(\tau)$ and take the integration by parts to obtain an estimate for $\dot{x}_{0}$ :

$$
\tilde{\dot{x}}_{0}=\int_{0}^{1} p_{4}(\tau) y(T \tau) d \tau .
$$

Finally, we get the estimates of $A$ and $\phi$ from the relation below by replacing $x(\tau)$ by $y(\tau)$ :

$$
A=\sqrt{x_{0}^{2}+\frac{\dot{x}_{0}^{2}}{\omega^{2}}}, \quad \phi=\arctan \left(\omega \frac{x_{0}}{\dot{x}_{0}}\right)
$$

\section{B. Study of the parameters influence on the estimation errors}

Let $y\left(t_{i}\right)=x\left(t_{i}\right)+C n\left(t_{i}\right)$ be a noisy measurement in the discrete case by taking $N$ equidistant zones at every period, where $x\left(t_{i}\right)=A \sin \left(\omega t_{i}+\phi\right), C \in \mathbb{R}, C>0$ and $n$ is a white noise with a standard normal distribution.

The trapezoidal rule applied to the four integral white noise parts of the proposition $\int_{0}^{1} p_{i}(\tau) n(T \tau) d \tau$ by taking $m+1$ points from the above sampling are given by

$$
Y_{i, m}=C h \sum_{l=1}^{m} \frac{p_{i}\left(t_{l}\right) n\left(T t_{l}\right)+p_{i}\left(t_{l-1}\right) n\left(T t_{l-1}\right)}{2}, i \in\{1,2,3,4\} \text {, }
$$

where $t_{l}=h . l$ and $h=1 / m$ for $l=1, \ldots, m$. If we assume that $\tilde{\omega}=\omega$ in $p_{3}\left(t_{l}\right)$ and $p_{4}\left(t_{l}\right)$, then each $Y_{i, m}$ is a finite sum of random independent Gaussian variables, so it's also a random Gaussian variable. Consequently, we can calculate their mean and their variance:

$$
\begin{aligned}
E\left[Y_{i, m}\right] & =h \sum_{l=1}^{m} \frac{p_{i}\left(t_{l}\right) E\left[n\left(T t_{l}\right)\right]+p_{i}\left(t_{l-1}\right) E\left[n\left(T t_{l-1}\right)\right]}{2} \\
& =0 . \\
\operatorname{Var}\left[Y_{i, m}\right] & =\frac{C^{2} h^{2}}{4}\left(p_{i}^{2}\left(t_{0}\right)+p_{i}^{2}\left(t_{m}\right)+4 \sum_{l=1}^{m-1} p_{i}^{2}\left(t_{l}\right)\right) .
\end{aligned}
$$

It's clear that

$$
\frac{Y_{i, m}}{\sqrt{\operatorname{Var}\left[Y_{i, m}\right]}} \sim \mathscr{N}(0,1)
$$

therefore, one gets the inequality below from the probability density function of a standard normal distribution:

$$
\left|Y_{i, m}\right| \stackrel{95.6 \%}{\leq} 2 \sqrt{\operatorname{Var}\left[Y_{i, m}\right]}=M_{2, i}(C, h, k, \mu, T)
$$

1) Error in the estimation of $\omega$ : Apply the trapezoidal rule to (1):

$$
\tilde{\omega}=\left(\frac{I_{1, m}+Y_{1, m}}{I_{2, m}+Y_{2, m}}\right)^{\frac{1}{2}} .
$$

where, for $i \in\{1,2\}$,

$$
I_{i, m}=\sum_{l=1}^{m} \frac{p_{i}\left(t_{l}\right) x\left(T t_{l}\right)+p_{i}\left(t_{l-1}\right) x\left(T t_{l-1}\right)}{2} h .
$$

The global error is a twofold error: one coming from the white noise part and the other one from the numerical integration approximation. They both depend on the parameters $k, \mu$ and $T$.

We denote the numerical integration approximation of $\omega$ by $\omega_{e}$ and by $e_{0}=\left|\tilde{\omega}^{2}-\omega_{e}^{2}\right|$ the absolute error which comes from the white noise in the estimation of $\omega^{2}$. We then have

Proposition 2: The global error in the estimation of $\omega^{2}$ can be bounded by:

$\left|\tilde{\omega}^{2}-\omega^{2}\right| \stackrel{95.6 \%}{\leq} M_{1}(C, h, k, \mu, T)+\left|\omega_{e}^{2}-\omega^{2}\right|=M(C, h, k, \mu, T)$ 
where

$$
\begin{aligned}
& M_{1}(C, h, k, \mu, T)=\frac{M_{2,5}(C, h, k, \mu, T)}{\left|I_{2, m}\right|\left(\left|I_{2, m}\right|-M_{2,2}(C, h, k, \mu, T)\right)}, \\
& \left|I_{2, m}\right|>M_{2,2}(C, h, k, \mu, T), \\
& M_{2,5}(C, h, k, \mu, T)=2 \sqrt{\operatorname{Var}\left[Y_{5, m}\right]}, \\
& Y_{5, m}=I_{2, m}, Y_{1, m}-I_{1, m} Y_{2, m} .
\end{aligned}
$$

Proof:

$e_{0}=\left|\tilde{\omega}^{2}-\omega_{e}^{2}\right|=\left|\frac{I_{1, m}+Y_{1, m}}{I_{2, m}+Y_{2, m}}-\frac{I_{1, m}}{I_{2, m}}\right|<\frac{\left|I_{2, m} Y_{1, m}-I_{1, m} Y_{2, m}\right|}{\left|I_{2, m}\right||| I_{2, m}|-| Y_{2, m}||}$.

Use (9) and if $\left|I_{2, m}\right|>M_{2,2}(C, h, k, \mu, T)$, we then have

$$
e_{0} \stackrel{95.6 \%}{\leq} \frac{\left|I_{2, m} Y_{1, m}-I_{1, m} Y_{2, m}\right|}{\left|I_{2, m}\right|\left(\left|I_{2, m}\right|-M_{2,2}(C, h, k, \mu, T)\right)} .
$$

Note $Y_{5, m}=I_{2, m} Y_{1, m}-I_{1, m} Y_{2, m}$ then it is also a random Gaussian variable. Consequently, we have $e_{0} \stackrel{95.6 \%}{\leq}$ $M_{1}(C, h, k, \mu, T)$. Finally,

$\left|\tilde{\omega}^{2}-\omega^{2}\right| \leq\left|\tilde{\omega}^{2}-\omega_{e}^{2}\right|+\left|\omega_{e}^{2}-\omega^{2}\right| \stackrel{95.6 \%}{\leq} M(C, h, k, \mu, T)$.

2) Error in the estimation of $x_{0}$ : Apply the trapezoidal rule to (7):

$$
\tilde{x}_{0}=\sum_{l=1}^{m} \frac{p_{3}\left(t_{l}\right) x\left(T t_{l}\right)+p_{3}\left(t_{l-1}\right) x\left(T t_{l-1}\right)}{2} h+Y_{3, m} .
$$

Proposition 3: The global error in the estimation of $x_{0}$ can be bounded by:

$$
\left|\tilde{x}_{0}-x_{0}\right| \stackrel{95.6 \%}{\leq} M_{2}(C, h, \mu, T)+\left|x_{0 e}-x_{0}\right|=M_{3}(C, h, \mu, T),
$$

where $h^{\prime}, k^{\prime}, \mu^{\prime}, T^{\prime}$ are the parameters that we have found out to estimate $\omega$ and

$$
\begin{aligned}
& M_{2}(C, h, \mu, T)=M\left(C, h^{\prime}, k^{\prime}, \mu^{\prime}, T^{\prime}\right) T^{2}|K|+\tilde{M}_{2,3}(h, \mu, T), \\
& K=\sum_{l=1}^{m} \frac{\left(1-t_{l}\right)^{\mu-1} t_{l} x\left(T t_{l}\right)+\left(1-t_{l-1}\right)^{\mu-1} t_{l-1} x\left(T t_{l-1}\right)}{2} h, \\
& \tilde{M}_{2,3}(C, h, \mu, T)=2 C \sqrt{\tilde{Z}(h, \mu, T)} \\
& \tilde{Z}(h, \mu, T)=h^{2} \sum_{l=1}^{m-1} \tilde{p}_{3}^{2}\left(t_{l}\right)+\frac{h^{2}}{4}\left(\tilde{p}_{3}^{2}\left(t_{0}\right)+\tilde{p}_{3}^{2}\left(t_{m}\right)\right), \\
& \tilde{p}_{3}\left(t_{l}\right)=|D|+T^{2}\left(1-t_{l}\right)^{\mu-1} t_{l}\left(\left|\omega_{e}^{2}\right|+M_{1}\left(C, h^{\prime}, k^{\prime}, \mu^{\prime}, T^{\prime}\right)\right) .
\end{aligned}
$$

proof:

Observe that $p_{3}\left(t_{l}\right)=D-\tilde{\omega}^{2} T^{2}\left(1-t_{l}\right)^{\mu-1} t_{l}$, where $D=$ $(\mu-1)\left(1-t_{l}\right)^{\mu-3}\left(2-\mu t_{l}\right)$. Then the numerical integration approximation of $x_{0}$ is given by

$$
\begin{aligned}
x_{0 e}= & \sum_{l=1}^{m} \frac{\left(D-\omega^{2} T^{2}\left(1-t_{l}\right)^{\mu-1} t_{l}\right) x\left(T t_{l}\right)}{2} h \\
& +\sum_{l=1}^{m} \frac{\left(D-\omega^{2} T^{2}\left(1-t_{l-1}\right)^{\mu-1} t_{l-1}\right) x\left(T t_{l-1}\right)}{2} h .
\end{aligned}
$$

The white noise error in the estimation of $x_{0}$ can be written as $\tilde{x}_{0}-x_{0 e}=\left(\omega^{2}-\tilde{\omega}^{2}\right) T^{2} K+Y_{3, m}$. Take the absolute value of $p_{3}\left(t_{l}\right)$ :

$$
\begin{aligned}
&\left|p_{3}\left(t_{l}\right)\right| \leq|D|+T^{2}\left(1-t_{l}\right)^{\mu-1} t_{l}\left|\tilde{\omega}^{2}\right| \\
& \stackrel{95.6 \%}{\leq}|D|+T^{2}\left(1-t_{l}\right)^{\mu-1} t_{l}\left(\left|\omega_{e}^{2}\right|+M_{1}\left(C, h^{\prime}, k^{\prime}, \mu^{\prime}, T^{\prime}\right)\right) \\
&=\tilde{p}_{3}\left(t_{l}\right)
\end{aligned}
$$

It yields a new bound of $Y_{3, m}$, which doesn't depend on $\tilde{\omega}$ :

$$
\begin{aligned}
& \left|Y_{3, m}\right| \stackrel{95.6 \%}{\leq} M_{2,3}(C, h, \mu, T) \quad(\text { depend on } \tilde{\omega}) \\
& \leq \quad \tilde{M}_{2,3}(C, h, \mu, T) \text {. }
\end{aligned}
$$

Consequently, we have

$$
\begin{array}{cl}
\left|\tilde{x}_{0}-x_{0 e}\right| & \stackrel{95.6 \%}{\leq} \\
\stackrel{95.6 \%}{\leq} & \left|\left(\omega^{2}-\tilde{\omega}^{2}\right)\right| T^{2}|K|+\tilde{M}_{2,3}(h, \mu, T) \\
& M_{2}(C, h, \mu, T) .
\end{array}
$$

Finally,

$$
\left|\tilde{x}_{0}-x_{0}\right| \stackrel{95.6 \%}{\leq} M_{2}(C, h, \mu, T)+\left|x_{0 e}-x_{0}\right|=M_{3}(C, h, \mu, T)
$$

3) Error in the estimation of $\dot{x}_{0}$ : Apply the trapezoidal rule to (9):

$$
\tilde{\dot{x}}_{0}=\sum_{l=1}^{m} \frac{p_{4}\left(t_{l}\right) x\left(T t_{l}\right)+p_{4}\left(t_{l-1}\right) x\left(T t_{l-1}\right)}{2} h+Y_{4, m} .
$$

Observe that $p_{4}\left(t_{l}\right)=E+\tilde{\omega}^{2} T\left(1-t_{l}\right)^{\mu-1}\left(1+(\mu-1) t_{l}\right)$, where $E=\frac{(\mu-1)(\mu-2)}{T}\left(1-t_{l}\right)^{\mu-3}-\frac{\mu-1}{T} D$. Then following the same reasonings as the one in the section $B-2)$, the following proposition can be stated:

Proposition 4: The global error in the estimation of $\dot{x}_{0}$ can be bounded by:

$$
\left|\tilde{\dot{x}}_{0}-\dot{x}_{0}\right| \stackrel{95.6 \%}{\leq} M_{4}(C, h, \mu, T)+\left|\check{\dot{x}}_{0}-\dot{x}_{0}\right|=M_{5}(C, h, \mu, T),
$$

where $\check{x}_{0}$ is the numerical integration approximation of $\dot{x}_{0}$ and

$$
\begin{aligned}
\left|\tilde{\dot{x}}_{0}-\check{\dot{x}}_{0}\right| \stackrel{95.6 \%}{\leq} & M\left(C, h^{\prime}, k^{\prime}, \mu^{\prime}, T^{\prime}\right)|T||V|+\tilde{M}_{2,4}(h, \mu, T) \\
= & M_{4}(C, h, \mu, T), \\
V & =\sum_{l=1}^{m} \frac{\left(1-t_{l}\right)^{\mu-1}\left(1+(\mu-1) t_{l}\right) x\left(T t_{l}\right)}{2} h \\
& +\sum_{l=1}^{m} \frac{\left(1-t_{l-1}\right)^{\mu-1}\left(1+(\mu-1) t_{l-1}\right) x\left(T t_{l-1}\right)}{2} h .
\end{aligned}
$$

\section{SOME MORE CALCULATIONS}

\section{A. New formulae}

Proposition 5: Let $g_{1}(t)$ and $g_{2}(t)$ be two continuous functions for $t \geq 0$, then the new estimations of $A$ and $\phi$ are given by

$$
\begin{aligned}
\tilde{A}^{2}= & T^{2} \frac{\left(F_{2}(T, \tilde{\omega}) P-G_{2}(T, \tilde{\omega}) Q\right)^{2}}{\left(G_{1}(T, \tilde{\omega}) F_{2}(T, \tilde{\omega})-G_{2}(T, \tilde{\omega}) F_{1}(T, \tilde{\omega})\right)^{2}} \\
& +T^{2} \frac{\left(G_{1}(T, \tilde{\omega}) Q-F_{1}(T, \tilde{\omega}) P\right)^{2}}{\left(G_{1}(T, \tilde{\omega}) F_{2}(T, \tilde{\omega})-G_{2}(T, \tilde{\omega}) F_{1}(T, \tilde{\omega})\right)^{2}}, \\
\tilde{\phi}= & \arctan \left(\frac{G_{1}(T, \tilde{\omega}) Q-F_{1}(T, \tilde{\omega}) P}{F_{2}(T, \tilde{\omega}) P-G_{2}(T, \tilde{\omega}) Q}\right)
\end{aligned}
$$

where

$$
\begin{gathered}
G_{1}(T, \tilde{\omega}) F_{2}(T, \tilde{\omega})-G_{2}(T, \tilde{\omega}) F_{1}(T, \tilde{\omega}) \neq 0, \\
P=\int_{0}^{1} g_{1}(T t) y(T t) d t, \quad Q=\int_{0}^{1} g_{2}(T t) y(T t) d t \\
G_{1}(T, \tilde{\omega})=\int_{0}^{T} g_{1}(t) \sin \tilde{\omega} t d t, \quad G_{2}(T, \tilde{\omega})=\int_{0}^{T} g_{1}(t) \cos \tilde{\omega} t d t,
\end{gathered}
$$


$F_{1}(T, \tilde{\omega})=\int_{0}^{T} g_{2}(t) \sin \tilde{\omega} t d t, \quad F_{2}(T, \tilde{\omega})=\int_{0}^{T} g_{2}(t) \cos \tilde{\omega} t d t$.

Proof: Take an expansion of $x$ :

$$
x(t)=A \sin \omega t \cdot \cos \phi+A \cos \omega t \cdot \sin \phi .
$$

Multiply both sides by two continuous functions $g_{1}(t)$ and $g_{2}(t)$. By integrating between 0 and $T$, one obtains:

$$
\begin{aligned}
& \int_{0}^{T} g_{1}(t) x(t) d t=A \cos \phi G_{1}(T, \omega)+A \sin \phi G_{2}(T, \omega) \\
& \int_{0}^{T} g_{2}(t) x(t) d t=A \cos \phi F_{1}(T, \omega)+A \sin \phi F_{2}(T, \omega) .
\end{aligned}
$$

It yields a linear system

$$
\left(\begin{array}{cc}
G_{1}(T, \omega) & G_{2}(T, \omega) \\
F_{1}(T, \omega) & F_{2}(T, \omega)
\end{array}\right)\left(\begin{array}{c}
A \cos \phi \\
A \sin \phi
\end{array}\right)=\left(\begin{array}{c}
L \\
U
\end{array}\right)
$$

where $L=\int_{0}^{T} g_{1}(t) x(t) d t$ and $U=\int_{0}^{T} g_{2}(t) x(t) d t$. Resolve the system by assuming that $G_{1}(T, \omega) F_{2}(T, \omega)-$ $G_{2}(T, \omega) F_{1}(T, \omega) \neq 0$

$$
\begin{aligned}
A \cos \phi & =\frac{F_{2}(T, \omega) L-G_{2}(T, \omega) U}{G_{1}(T, \omega) F_{2}(T, \omega)-G_{2}(T, \omega) F_{1}(T, \omega)}, \\
A \sin \phi & =\frac{G_{1}(T, \omega) U-F_{1}(T, \omega) L}{G_{1}(T, \omega) F_{2}(T, \omega)-G_{2}(T, \omega) F_{1}(T, \omega)} .
\end{aligned}
$$

The proof can be completed by replacing $x(t)$ by $y(t)$.

B. Study of the parameters influence on the estimation errors

Apply the trapezoidal rule to the estimation of $A$ and $\phi$ :

$$
\begin{aligned}
\tilde{A}^{2}= & T^{2} \frac{\left(F_{2}(T, \tilde{\omega}) P_{m}-G_{2}(T, \tilde{\omega}) Q_{m}\right)^{2}}{\left(G_{1}(T, \tilde{\omega}) F_{2}(T, \tilde{\omega})-G_{2}(T, \tilde{\omega}) F_{1}(T, \tilde{\omega})\right)^{2}} \\
& +T^{2} \frac{\left(G_{1}(T, \tilde{\omega}) Q_{m}-F_{1}(T, \tilde{\omega}) P_{m}\right)^{2}}{\left(G_{1}(T, \tilde{\omega}) F_{2}(T, \tilde{\omega})-G_{2}(T, \tilde{\omega}) F_{1}(T, \tilde{\omega})\right)^{2}}, \\
\tilde{\phi}= & \arctan \left(\frac{G_{1}(T, \tilde{\omega}) Q_{m}-F_{1}(T, \tilde{\omega}) P_{m}}{F_{2}(T, \tilde{\omega}) P_{m}-G_{2}(T, \tilde{\omega}) Q_{m}}\right) .
\end{aligned}
$$

Observe that $P_{m}=L_{m}+X_{1, m}$ and $Q_{m}=U_{m}+X_{2, m}$, where the white noise parts are given by

$$
X_{i, m}=C h \sum_{l=1}^{m} \frac{g_{i}\left(t_{l}\right) n\left(T t_{l}\right)+g_{i}\left(t_{l-1}\right) n\left(T t_{l-1}\right)}{2}, i \in\{1,2\} \text {. }
$$

1) Error in the estimation of $A$ : Assume that $\tilde{\omega}=\omega$ and denote by $A_{e}$ the numerical integration approximation of $A$, such that the estimation error of $A^{2}$ is given by

Proposition 6: The global error in the estimation of $A^{2}$ can be bounded by:

$$
\left|\tilde{A}^{2}-A^{2}\right| \stackrel{95.6 \%}{\leq} M_{6}(C, h, T)+\left|A_{e}^{2}-A^{2}\right|=M_{7}(C, h, T),
$$

where

$$
\begin{aligned}
& M_{6}(C, h, T)= \frac{T^{2}\left(3 \operatorname{Var}\left[X_{3, m}\right]+4 \sqrt{\operatorname{Var}\left[X_{5, m}\right]}+3 \operatorname{Var}\left[X_{4, m}\right]\right)}{\left(G_{1}(T, \tilde{\omega}) F_{2}(T, \tilde{\omega})-G_{2}(T, \tilde{\omega}) F_{1}(T, \tilde{\omega})\right)^{2}}, \\
& X_{3, m}=F_{2}(T, \tilde{\omega}) X_{1, m}-G_{2}(T, \tilde{\omega}) X_{2, m}, \\
& X_{4, m}=G_{1}(T, \tilde{\omega}) X_{2, m}-F_{1}(T, \tilde{\omega}) X_{1, m} \\
& X_{5, m}=J_{1, m} X_{3, m}+J_{2, m} X_{4, m}, \\
& J_{1, m}=F_{2}(T, \tilde{\omega}) L_{m}-G_{2}(T, \tilde{\omega}) U_{m}, \\
& J_{2, m}=G_{1}(T, \tilde{\omega}) U_{m}-F_{1}(T, \tilde{\omega}) L_{m} .
\end{aligned}
$$

proof: Observe that

$$
\begin{aligned}
& \left(F_{2}(T, \tilde{\omega}) P_{m}-G_{2}(T, \tilde{\omega}) Q_{m}\right)^{2}=J_{1, m}^{2}+X_{3, m}^{2}+2 J_{1, m} X_{3, m}, \\
& \left(G_{1}(T, \tilde{\omega}) Q_{m}-F_{1}(T, \tilde{\omega}) P_{m}\right)^{2}=J_{2, m}^{2}+X_{4, m}^{2}+2 J_{2, m} X_{4, m} .
\end{aligned}
$$

Then the white noise error in the estimation of $A^{2}$ is given by

$$
\tilde{A}^{2}-A_{e}^{2}=\frac{T^{2}\left(X_{3, m}^{2}+2 X_{5, m}+X_{4, m}^{2}\right)}{\left(G_{1}(T, \tilde{\omega}) F_{2}(T, \tilde{\omega})-G_{2}(T, \tilde{\omega}) F_{1}(T, \tilde{\omega})\right)^{2}} .
$$

Since $\frac{X_{i, m}}{\sqrt{\operatorname{Var}\left[X_{i, m}\right]}} \sim \mathscr{N}(0,1), i \in\{3,4\}$, it comes that $\frac{X_{i, m}^{2}}{\operatorname{Var}\left[X_{i, m}\right]}$ follows the $\chi^{2}$ distribution with the probability density function $f_{X}(t)=\frac{\sqrt{2}}{2 \Gamma(1 / 2)} t^{-\frac{1}{2}} e^{-\frac{t}{2}}, \Gamma(1 / 2)=\int_{0}^{+\infty} t^{-\frac{1}{2}} \exp (-t) d t$. Consequently, $X_{i, m}^{2} \stackrel{95.6 \%}{\leq} 3 \operatorname{Var}\left[X_{i, m}\right]$, and the proof can be completed by using $\left|X_{5, m}\right| \stackrel{95.6 \%}{\leq} 2 \sqrt{\operatorname{Var}\left[X_{5, m}\right]}$.

2) Error in the estimation of $\phi$ : Observe that

$$
\tan (\tilde{\phi})=\frac{J_{2, m}+X_{4, m}}{J_{1, m}+X_{3, m}} .
$$

Assume that $\tilde{\omega}=\omega$ and denote by $\phi_{e}$ the numerical integration approximation of $\phi$, then

Proposition 7: The global error in the estimation of $\tan (\phi)$ can be bounded by:

$$
\begin{aligned}
|\tan (\tilde{\phi})-\tan (\phi)| \stackrel{95.6 \%}{\leq} & M_{8}(C, h, T)+\left|\tan \left(\phi_{e}\right)-\tan (\phi)\right| \\
& =M_{9}(C, h, T),
\end{aligned}
$$

where

$$
\begin{array}{r}
M_{8}(C, h, T)=\frac{2 \sqrt{\operatorname{Var}\left[X_{6, m}\right]}}{\left|J_{1, m}\right|\left(\left|J_{1, m}\right|-2 \sqrt{\operatorname{Var}\left[X_{3, m}\right]}\right)}, \\
\left|J_{1, m}\right|>2 \sqrt{\operatorname{Var}\left[X_{3, m}\right]}, \quad X_{6, m}=J_{1, m} X_{4, m}-J_{2, m} X_{3, m} .
\end{array}
$$

proof: The white noise error in the estimation of $\tan (\phi)$ is given by

$$
\begin{aligned}
&\left|\tan (\tilde{\phi})-\tan \left(\phi_{e}\right)\right|=\left|\frac{J_{2, m}+X_{4, m}}{J_{1, m}+X_{3, m}}-\frac{J_{2, m}}{J_{1, m}}\right| \\
& \stackrel{95.6 \%}{\leq} \frac{\left|J_{1, m} X_{4, m}-J_{2, m} X_{3, m}\right|}{\left|J_{1, m}\right|\left(\left|J_{1, m}\right|-2 \sqrt{\operatorname{Var}\left[X_{3, m}\right]}\right)} \\
& \stackrel{95.6 \%}{\leq} M_{8}(C, h, T) .
\end{aligned}
$$$$
95.6 \%
$$

Finally, $|\tan (\tilde{\phi})-\tan (\phi)| \stackrel{95.6 \%}{\leq} M_{9}(C, h, T)$.

\section{EXEMPLE}

Take $\omega=1, A=1, \phi=\pi / 4, C=0.1$ and $N=100$, so $h_{s}=$ $2 \pi \times 10^{-2}$. Each estimation is a random variable. In order to minimize the noise corruption, we then take 200 tests of the inequality about the estimation error in Proposition 2 (resp. Proposition 3 and Proposition 4) in Figure 1 (resp. Figure 2 and Figure 3) with the parameters where $M(C, h, k, \mu, T)$ (resp. $M_{3}(C, h, \mu, T)$ and $M_{5}(C, h, \mu, T)$ ) get its minimum . Using Proposition 1, the relative estimation errors of $A$ and $\phi$ are shown in Figure 4 and 5 respectively. In order to find out the minimum of $M_{3}(C, h, \mu, T)$, we fix the value 
of $\mu$. We find the corresponding minimum by varying $m$ $\left(T=h_{s} m\right)$ and we change the value of $\mu$. We then compute the corresponding minimum value. The results are shown in the table below:

\begin{tabular}{|c|c|c|}
\hline Value & $m$ & Minimum \\
\hline$\mu=3$ & 28 & 0.0924 \\
\hline$\mu=4$ & 38 & 0.0884 \\
\hline$\mu=5$ & 48 & 0.0877 \\
\hline$\mu=6$ & 59 & 0.0874 \\
\hline$\mu=7$ & 72 & 0.0872 \\
\hline$\mu=8$ & 82 & 0.0871 \\
\hline$\mu=9$ & 98 & 0.0869 \\
\hline$\mu=10$ & 110 & 0.0868 \\
\hline
\end{tabular}

We can see that the minimum is slightly decreasing as $\mu$ and $m$ increase. We take $\mu=10$ and $T=110 h_{s}$ to estimate $x_{0}$. Similarly, a parameter estimation for $\dot{x}_{0}$ has been made.

In order to find out the minimum of $M(C, h, k, \mu, T)$, first we fix the value of $k$ and we find the corresponding minimum that depends on $\mu$ and $m$ with the same way as above. We then change the value of $k$ to find the corresponding minimum. According to the table below, we take $k=6, \mu=6$ and $T=115 h_{s}$ to estimate $\omega$.

\begin{tabular}{|c|c|c|c|}
\hline Value & $\mu$ & $m$ & Minimum \\
\hline$k=2$ & 3 & 110 & 0.0603 \\
\hline$k=3$ & 4 & 113 & 0.0470 \\
\hline$k=4$ & 4 & 107 & 0.0398 \\
\hline$k=5$ & 5 & 111 & 0.0380 \\
\hline$k=6$ & 6 & 115 & 0.0377 \\
\hline$k=7$ & 8 & 125 & 0.0379 \\
\hline$k=8$ & 10 & 134 & 0.0384 \\
\hline$k=9$ & 9 & 113 & 0.0407 \\
\hline
\end{tabular}

We take $g_{1}(t)=\exp (t)$ and $g_{2}(t)=\exp (2 t)$ in Proposition 5 and we take 200 tests of the inequality of the estimation error in Proposition 6 (resp. Proposition 7) in Figure 6 (resp. Figure 7) with the parameters where $M_{7}(C, h, T)$ (resp. $M_{9}(C, h, T)$ ) get its minimum. The minimum is found out with the same way as above. Using Proposition 5, the relating estimation errors of $A$ and $\phi$ are shown in Figure 7 and in Figure 9.

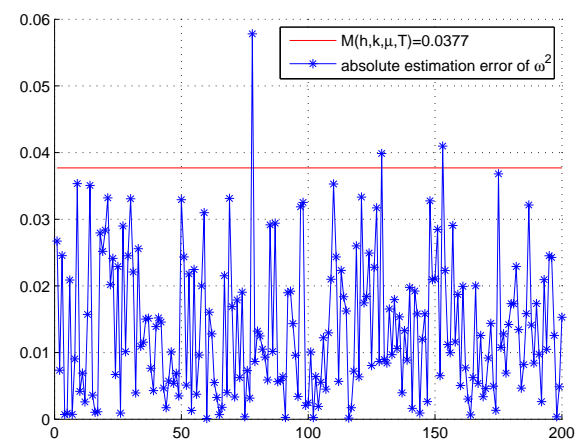

Fig. 1. $\left|\tilde{\omega}^{2}-\omega^{2}\right|$ and $M(C, h, k, \mu, T)$ with $k=6, \mu=6$ and $T=1.15 \times 2 \pi$

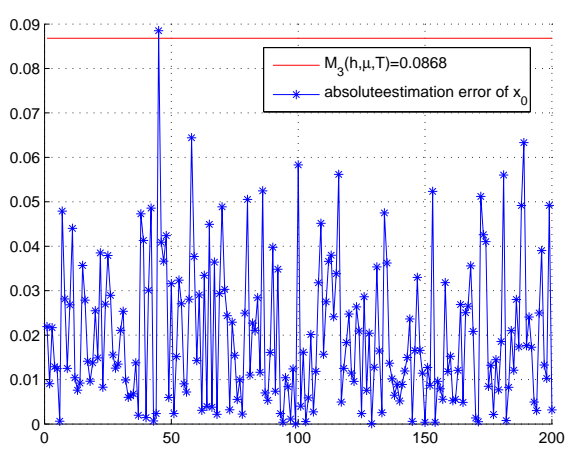

Fig. 2. $\left|\tilde{x}_{0}-x_{0}\right|$ and $M_{3}(C, h, \mu, T)$ with $\mu=10$ and $T=1.10 \times 2 \pi$

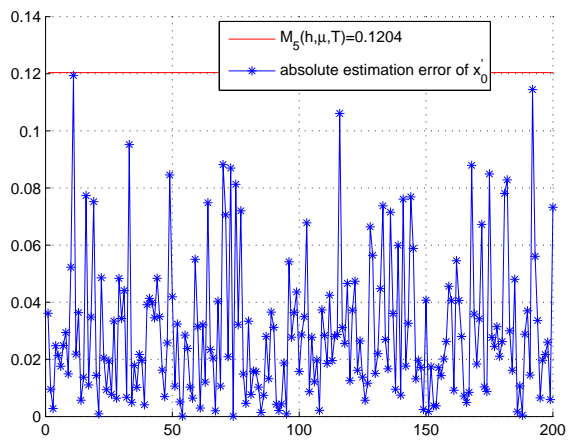

Fig. 3. $\left|\tilde{\dot{x}}_{0}-\dot{x}_{0}\right|$ and $M_{5}(C, h, \mu, T)$ with $\mu=3$ and $T=1.14 \times 2 \pi$

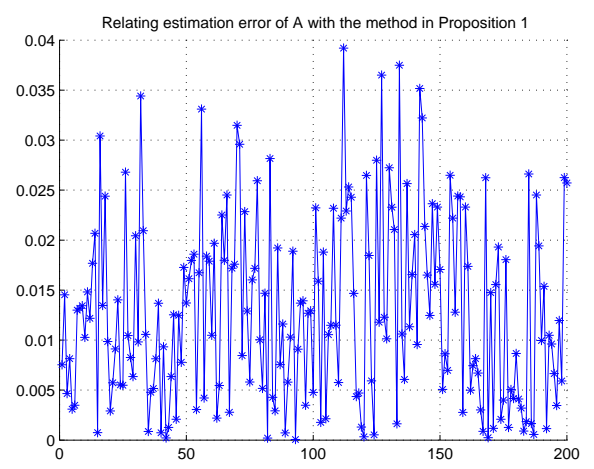

Fig. 4. Relating estimation error of $A$ with the method in Proposition1

\section{CONCLUSION}

The same type of analysis will be given in some future publications for other types of noises, such as Poisson white noises or high frequency sinusoids. Moreover, the BienayméChebyshev inequality will be considered so as to obtain a bound on the estimation error for any kind of noise.

\section{REFERENCES}

[1] M. Fliess, Analyse non standard du bruit, C.R. Acad. Sci. Paris Ser. I, 342 (2006) 797-802.

[2] M. Fliess, Critique du rapport signal à bruit en théorie de l'information, Manuscript, 2007 (available at http://hal.inria.fr/inria-00195987/en/). 


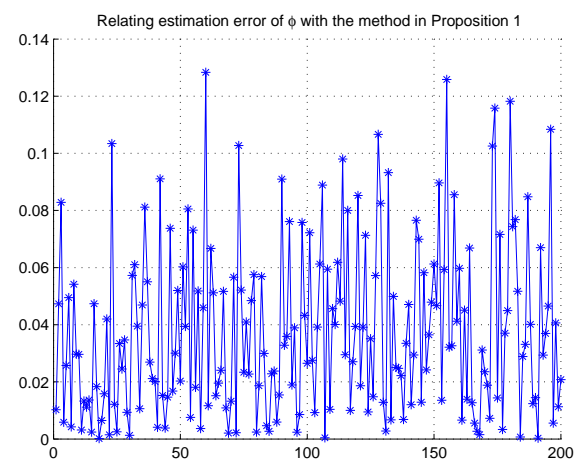

Fig. 5. Relating estimation error of $\phi$ with the method in Proposition1

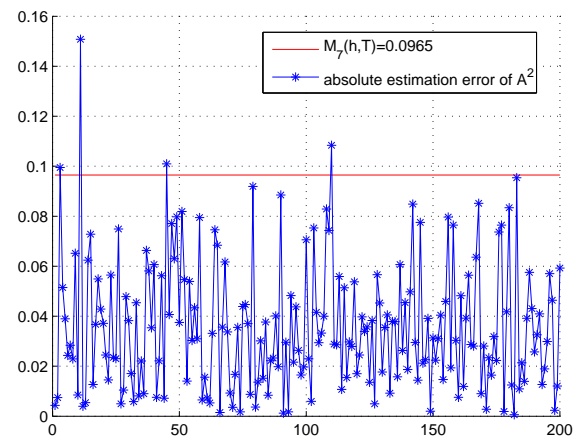

Fig. 6. $\left|\tilde{A}^{2}-A^{2}\right|$ and $M_{7}(C, h, T)$ with $T=0.83 \times 2 \pi$ by taking $\tilde{\omega}=\omega$

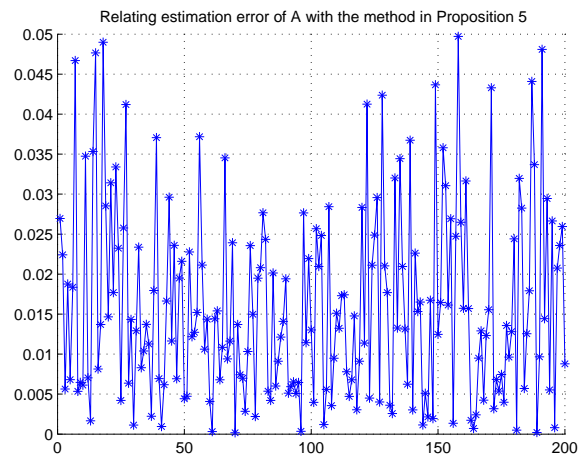

Fig. 7. Relating estimation error of $A$ with the method in Proposition 5

[3] M. Fliess, M. Mboup, H. Mounier, H. Sira-Ramírez, Questioning some paradigms of signal processing via concrete examples, in Algebraic Methods in Flatness, Signal Processing and State Estimation, H. Sira-Ramírez, G. Silva-Navarro (Eds.), Editiorial Lagares, México, 2003, pp. 1-21 (available at http://hal.inria.fr/inria-00001059/en/).

[4] M. Fliess, H. Sira-Ramírez, An algebraic framework for linear identification, ESAIM Control Optim. Calc. Variat., 9 (2003) 151-168.

[5] M. Fliess, H. Sira-Ramírez, Closed-loop parametric identification for continuous-time linear systems via new algebraic techniques, in H. Garnier, L. Wang (Eds): Identification of Continuous-time Models from Sampled Data, pp. 363-391,, Springer, 2008 (available at http://hal.inria.fr/inria-00114958/en/).

[6] M. Mboup, Parameter estimation via differential algebra and operational calculus, Manuscript, 2006 (available at http://hal.inria.fr/inria-00138294).

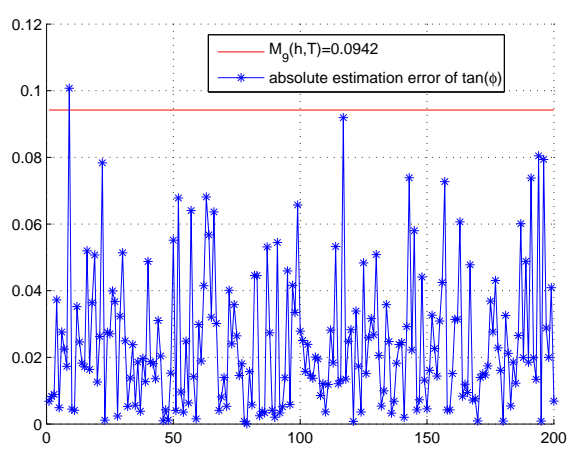

Fig. 8. $|\tan (\tilde{\phi})-\tan (\phi)|$ and $M_{9}(C, h, T)$ with $T=0.57 \times 2 \pi$ by taking $\tilde{\omega}=\omega$

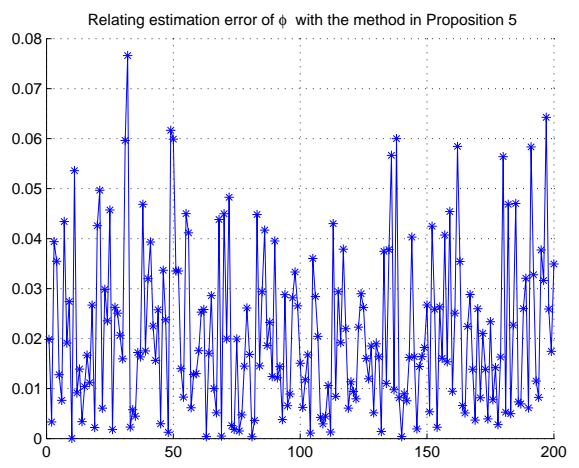

Fig. 9. Relating estimation error of $\phi$ with the method in Proposition 5

[7] A. Neves, M. Mboup, M. Fliess, An Algebraic Receiver for Full Response CPM Demodulation, VI INTERNATIONAL TELECOMMUNICATIONS SYMPOSIUM (ITS2006), SEPTEMBER 3-6, 2006, FORTALEZA-CE, BRAZIL.

[8] A. Neves, M.D. Miranda, M. Mboup, Algebraic parameter estimation of damped exponentials, Proc. 15 $5^{\text {th }}$ Europ. Signal Processing Conf. - EUSIPCO 2007, Poznań, 2007 (available at http://hal.inria.fr/inria-00179732/en/.

[9] J.G. Proakis, Digital Communications, $4^{\text {th }}$ ed., McGraw-Hill, 2001.

[10] J.R. Trapero, H. Sira-Ramírez, V.F. Battle, An algebraic frequency estimator for a biased and noisy sinusoidal signal, Signal Processing, 87 (2007) 1188-1201.

[11] J.R. Trapero, H. Sira-Ramírez, V.F. Battle, A fast on-line frequency estimator of lightly damped vibrations in flexible structures, J. Sound Vibration, 307 (2007) 365-378.

[12] J.R. Trapero, H. Sira-Ramírez, V.F. Battle, On the algebraic identification of the frequencies, amplitudes and phases of two sinusoidal signals from their noisy sums, Int. J. Control, 81 (2008) 505-516. 